\title{
EDITORIAL
}

\section{Cardioversion and the use of sedation}

\author{
S J Harrison, J Mayet
}

Heart 2004:90:1374-1376. doi: 10.1136/hrt.2004.040113

Nurse led cardioversion services have achieved significant reductions in both cost and waiting time. However, the question of safety of the procedure raises several areas of concern

See end of article for authors' affiliations

....................

Correspondence to: Dr Sally J Harrison, Royal Free Hospital, London, UK; sallyharrison@doctors. org.uk
$\mathrm{P}$ atients with persistent atrial fibrillation requiring direct cardioversion present a significant challenge to the cardiology department. Traditionally a cardioversion required the coordination of a starved patient with four members of staff: cardiologist, cardiology nurse, anaesthetist, and an anaesthetic assistant. Coordination of this team can be potentially difficult as this short procedure is often scheduled on an ad hoc basis with no dedicated theatre slot; this may result in frequent cancellations, poor patient satisfaction, and a reduced chance of successful cardioversion. A number of groups have addressed this by not involving the anaesthetist (and anaesthetic assistant) and have proceeded with physician led sedation for cardioversion. ${ }^{12}$ The European working time directive and economic constraints have further reduced the number of doctors at work during normal working hours.

The deficit of doctors has been met in part by specialist nurses expanding their roles to cover tasks previously fulfilled by junior doctors. This change in approach has been embraced by Boodhoo and colleagues ${ }^{3}$ who describe in this issue of Heart their experiences of a nurse led cardioversion service. They have reduced the number of staff involved by replacing the anaesthetist and cardiologist with two carefully selected specialist nurses with coronary care unit experience and certificates of attendance for ALS and safe sedation practice. They have achieved significant reductions in both cost and waiting time to cardioversion. The success of the cardioversion was comparable to other groups at three months; however the question of safety of the procedure raises several areas of concern.

\section{DEEP SEDATION HAS A NARROW MARGIN OF SAFETY}

External cardioversion is a short, painful procedure with a stimulus intensity similar to that of a surgical incision. The level of sedation required for cardioversion is either "deep sedation" or general anaesthesia. Adequate depths of sedation are important to firstly prevent recall of an unpleasant experience and secondly to attenuate the catecholamine surge of the stress response. This is particularly important in a patient population with a high rate of myocardial ischaemia and when rhythm stabilisation is the ultimate goal. The levels of sedation described by Boodhoo and colleagues ${ }^{3}$ would be classified as moderate to deep sedation (that is, responding to tactile or painful stimulus). They report no significant respiratory events, or need for reversal of over-sedation with flumazanil, but report a recall rate of 1 in 50. This superficially appears to be a good result; however, general anaesthesia has a reported awareness with recall rate of less than $0.3 \%$ and an excellent safety record with mortality rates less than $\mathrm{l}$ in $185000 .{ }^{4}$ Their study of 394 procedures is underpowered to make any claims of safety.

It could be argued that it is safer to have patients recalling the procedure than having respiratory depression, but this highlights the problem that the depth of sedation needed for cardioversion has to strike a fine and somewhat difficult balance-firstly, to prevent recall and attenuate stress responses; and secondly, to not precipitate hypoventilation, airway obstruction, or cardiac embarrassment. The Royal College of Anaesthetists are very clear in their guidelines for implementing and ensuring safe sedation practice: if verbal responsiveness is lost the patient requires a level of care identical to that needed for general anaesthesia. ${ }^{5}$ In the report in this journal a doctor (presumably a cardiologist) was available in the next room. This doctor would no doubt be excellent at managing poor cardiac output states and rhythm disturbances, but is likely to lack the airway skills to adequately maintain an airway in a hypoxic over-sedated patient. This situation can challenge an experienced anaesthetist (and assistant), which is why anaesthetists often prefer the more predictable and controlled position of a short general anaesthetic than deep sedation for this type of procedure. It is recommended that an anaesthetist works with a skilled assistant (operating department assistant), especially when working in isolated areas away from the main theatre complex. When airway problems occur, they deteriorate rapidly to hypoxia and cardiac arrest if not dealt with quickly. Two pairs of hands trained in airway skills are often needed to retrieve such a situation.

\section{PATIENT SELECTION}

Many of the lessons learnt in physician led sedation have come from the gastroenterologists. In their guidelines for sedation they suggest the assistance of an anaesthetist if the patient has the potential for deep sedation together with one or more sedation related risk factors. ${ }^{6}$ The latter includes patients with predicted difficult airways and significant medical conditions. Many patients with atrial fibrillation will classify as American Society of Anesthesiologists (ASA) III; 
that is a patient with severe systemic disease that is not incapacitating. It is known that ASA III patients tolerate adverse events poorly and have a higher surgical mortality. ${ }^{7}$ Of particular concern is that a cardiorespiratory event secondary to over-sedation may be poorly tolerated in these patients. Furthermore, a cardiac event secondary to a primary airway problem that is not recognised could wrongly be attributed to the patient's poor baseline cardiac status. This could lead to a falsely low reporting of sedation related events.

Predicting potentially difficult airways is an important requirement of any team involved with deeply sedating patients. The ASA guidelines have compiled a useful list of such patients, and it must be stressed that the most common subgroup of these are obese patients. ${ }^{8}$ Drug handling is unpredictable in obese patients; even in the absence of obstructive sleep apnoea they are often difficult to hand ventilate with a bag and mask, and difficult to intubate with endotracheal tubes. ${ }^{9}$ Any group offering moderate to deep sedation should be very cautious when including obese patients. Boodhoo and colleagues ${ }^{3}$ point out that it was not possible to deeply sedate some obese patients using $40 \mathrm{mg}$ of diazepam.

\section{PHARMACOLOGY}

The ideal pharmacological agent to produce adequate deep sedation or anaesthesia for cardioversion does not exist. Rapid onset and offset, cardiovascular stability, no respiratory depression, and some analgesic component would be the ideal characteristics. The most common agents used by nonanaesthetists are benzodiazepines with or without opiate supplementation. Diazepam and midazolam have both been used successfully for cardioversion, and which agent is the better still remains unclear. ${ }^{10}$ Compared with anaesthetic induction agents, benzodiazepines are a poor choice of agent to provide deep sedation or anaesthesia, because the large doses required lead to prolonged sedative effects, with the associated risks of respiratory depression in the recovery period.

Boodhoo and colleagues ${ }^{3}$ describe a third of their patients as having sedative side effects at 24 hours and $17 \%$ at 48 hours using diazepam. This group have described better experiences in terms of recovery with diazepam rather than midazolam. This is contrary to what one might expect as midazolam has a shorter half life. Midazolam has become more popular for use by the gastroenterologists, but their procedures differ in that they tend to require lighter sedation and are of longer duration than cardioversions. Some groups have claimed that midazolam with routine reversal by flumazenil to be a favoured technique for cardioversion, but others have suggested an incidence of re-sedation after four hours despite benzodiazepine reversal. ${ }^{11}$ Many groups have used opiates in combination with benzodiazepines successfully, and intuitively giving analgesia for a painful procedure makes sense. The safe sedation guidelines caution using synergistic agents as the safety margin between "conscious sedation" and "anaesthesia" can be significantly reduced. Since deep sedation/anaesthesia is required for cardioversion it is debatable whether it is safer to achieve this with a single benzodiazepine or using polypharmacy. In terms of ensuring safe, clear guidelines for nurse led sedation a single agent would only require a single reversal agent (that is, flumazenil), which is safer in the event of over-sedation.

A survey of anaesthetists in 2003 showed that the most commonly used agent (90\%) was propofol, with 9\% using etomidate, and $43 \%$ additionally using a short acting opiate. ${ }^{12}$ Propofol is short acting, so ideal for day cases, but requires airway manipulation of the patient when used for deep sedation or anaesthesia (so not appropriate for use by the non-anaesthetist for cardioversion). The inhalational volatile agent sevoflurane has a rapid onset and offset of action with probably greater cardiovascular stability for cardioversion than propofol and is being used increasingly by anaesthetists. ${ }^{13}$ It again requires skilled airway manipulation of the patient. Most sedatives and anaesthetic induction agents cause hypotension by a decrease in systemic vascular resistance (at least in part). Patients with very poor cardiac function can become hypotensive before adequate levels of sedation are achieved, and judicious titration of vasoconstrictors such as ephedrine, metaraminol, or phenylephrine are useful adjuncts for supporting the circulation during these short procedures.

\section{MONITORING OF DEEP SEDATION}

The traditional monitoring for conscious sedation has been non-invasive blood pressure, pulse oximetry, patient observation, and maintaining verbal contact with the patient. The minimal monitoring requirements for general anaesthesia also includes ECG and capnography. ${ }^{14}$ It must be stressed that pulse oximetry alone is a poor monitor of the adequacy of ventilation of a patient. A patient who has supplementary oxygen can easily be maintaining their oxygen levels but grossly hypoventilating and so retaining carbon dioxide to dangerous levels. The serious consequences of this are arrhythmias, somnolence, and coma leading to further respiratory depression, seizures, and cardiorespiratory arrest. For these reasons, when respiratory depression becomes increasingly likely, it is important to measure end tidal carbon dioxide levels as well as oxygen saturation. When deeply sedating patients for cardioversion using long acting agents (such as benzodiazepines) it could be argued that there is a place for the routine use of capnography. Several groups of non-anaesthetists have described early recognition of respiratory depression with the use of capnography in deeply sedated patients. ${ }^{15} 16$

\section{CONCLUSION}

The gold standard for cardioversion is a brief period of general anaesthesia or deep sedation using short acting agents with a rapid recovery profile. It requires the presence of an airway specialist (that is, an anaesthetist) to provide controlled airway support and maintenance of the cardiac output if required. A significant cost reduction and a shorter waiting time has been demonstrated by using nurse led sedation, but it is likely that the patients are being exposed to unnecessary risks. The patient population for cardioversion are not straightforward to deeply sedate or anaesthetise, as they have co-existent medical problems and may have a reduced cardiac output after the procedure. ${ }^{17}$ The study by Boodhoo and colleagues ${ }^{3}$ is underpowered to demonstrate safety of a nurse led strategy. Patient selection is crucial and obese patients and patients whose status is ASA III should certainly be managed by airway specialists. The use of capnography could improve early identification of respiratory depression and a combination of this, adequate patient selection, and very close cover should be considered by any department thinking about setting up such a system. Careful auditing of any new approach is essential and it has been suggested that a national confidential enquiry into sedation induced adverse events may be the only way to truly assess the safety of non-anaesthetist deep sedation. ${ }^{18}$

\footnotetext{
Authors' affiliations

S J Harrison, Royal Free Hospital, London, UK

J Mayet, International Centre for Circulatory Health, St Mary's Hospital and Imperial College, London, UK
} 


\section{REFERENCES}

1 Pugh PJ, Spurrell P, Kamalvand K, et al. Sedation by physician with diazepam for DC cardioversion of atrial arrhythmias. Heart 2001;86:572-3.

2 Raipancholia R, Sentinella L, Lynch M. Role for conscious sedation for external cardioversion. Heart 2001;86:571-2.

3 Boodhoo L, Bordoli G, Mitchell A, et al. The safety and effectiveness of a nurse led cardioversion service under sedation. Heart 2004;90:1443-6.

4 Jenkins K, Baker AB. Consent and anaesthetic risk. Anaesthesia 2003;58:962-84.

5 Royal College of Anaesthetists. Implementing and ensuring safe sedation practice for healthcare procedures in adults. Report of a working Party established by the Royal College of Anaesthetists, www.aomrc.org.uk November 2001.

6 American Society for Gastroinestinal Endoscopy. Sedation and monitoring of patients undergoing gastrointestinal endoscopic procedures. Gastrointest Endosc 1995:42:626-9.

7 Vacanti CJ, Van Houten RJ, Hill RC. A statistical analysis of the relationship of physical status to postoperative mortality in 68388 cases. Anesth Analg 1970;49:564.

8 American Society of Anesthesiologists. Practice guidelines for sedation and analgesia by nonanesthesiologists. An updated report by the ASA task force on sedation and analgesia by nonanesthesiologists. Anesthesiology 2002;96:1004-17.
9 Adams JP, Murphy PJ. Obesity in anaesthesia and intensive care. $\mathrm{Br} J$ Anaesth 2000;85:91-108.

10 Stoneham MD. Anaesthesia for cardioversion. Anaesthesia 1996:51:565-70.

11 Fennelly ME, Powell H, Galletly DC, et al. Midazolam sedation reversed with flumazenil for cardioversion. Br J Anaesth 1992;68:303-5.

12 James S, Broome IJ. Anaesthesia for cardioversion. Anaesthesia 2003:58:291-2.

13 Karthikeyan S, Balachandran S, Cort J, et al. Anaesthesia for cardioversion: a comparison of sevoflurane and propofol. Anaesthesia 2002;57:1114-9

14 Association of Anaesthetists of Great Britain and Ireland. Recommendations for Standards of Monitoring During Anaesthesia and Recovery. Revised December 2000.

15 Vargo JJ, Zuccaro G, Dumont JA, et al. Automated graphic assessment of respiratory activity is superior to pulse oximetry and visual assessment for the detection of early respiratory depression during therapeutic upper endoscopy. Gastrointest Endoscopy 2002;55:826-31.

16 Mcquillan KK, Steele DW. Capnography during sedation/analgesia in the pediatric emergency department. Pediatr Emerg Care 2000;16:401-4.

17 Upshaw CB. Hemodynamic changes after cardioversion of chronic atrial fibrillation. Arch Intern Med 1997;157:1004-17.

18 Lawson GR. Sedation of children for magnetic resonance imaging. Arch Dis Child 2000;82:150-3.

\section{IMAGES IN CARDIOLOGY}

\section{Coconut atrium following thoracic radiotherapy}

A 45 year old man who had previously undergone curative surgery and radiotherapy for a thoracic neuroblastoma in childhood was referred for assessment of breathlessness and exertional dizziness. A high resolution thoracic computed tomographic scan demonstrated patchy pulmonary fibrosis and incidentally revealed the presence of localised extensive calcification throughout the left atrium (panel A). He subsequently underwent cardiac catheterisation and fluoroscopy confirmed the presence of gross calcification affecting most of the left atrium (panel B, right anterior oblique view). Angiography revealed normal coronary arteries and left ventricular function with no evidence of mitral regurgitation. The pulmonary artery pressure was 53/ $12 \mathrm{~mm} \mathrm{Hg}$, with a mean of $34 \mathrm{~mm} \mathrm{Hg}$, and the mean pulmonary capillary wedge pressure was $20 \mathrm{~mm} \mathrm{Hg}$ giving a transpulmonary gradient of $14 \mathrm{~mm} \mathrm{Hg}$. Strikingly, the phasic pressure tracing demonstrated giant $\mathrm{v}$ waves despite the absence of significant mitral regurgitation or a raised left ventricular end diastolic pressure (post a wave $12 \mathrm{~mm} \mathrm{Hg}$ ) (panel C). These findings indicated left atrial non-compliance caused by calcification, suggesting that his symptoms were at least in part caused by a rapid increase in left atrial pressure with exercise. This condition, known as "porcelain" or "coconut" atrium, is rare and can occur in
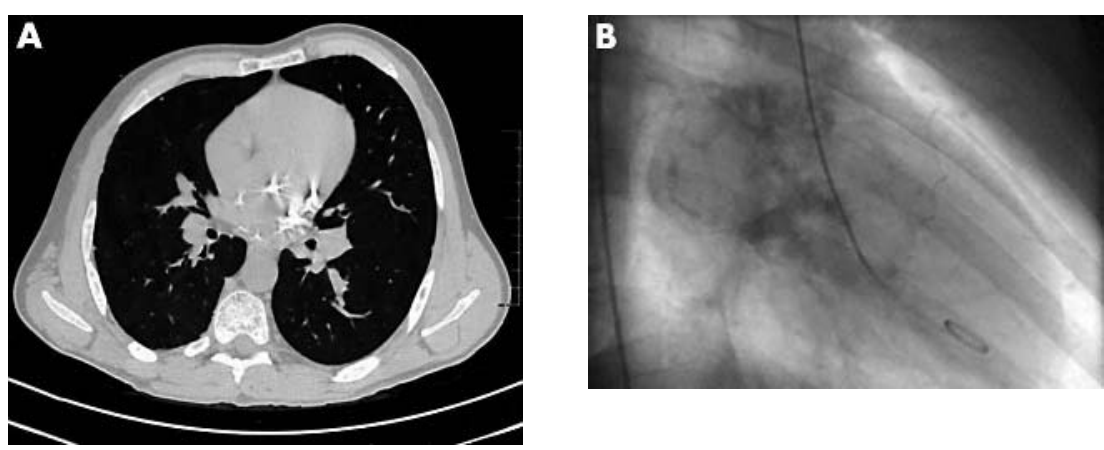

patients with rheumatic mitral valve disease, but has not previously been described in relation to thoracic radiotherapy. Surgical resection by endoatriectomy may occasionally be an option when calcification is nontransmural and spares the septum. Patients may be sensitive to altered loading conditions relating to diuretic or vasodilator treatment, and may be at increased risk of thromboembolism.

N P Jenkins N H Brooks M Greaves npjenkins@doctors.org.uk 